\title{
Effective Protection for Automotive Electronics from the Electromagnetic Interference
}

\author{
Tamilarasu. $\mathrm{S}^{1}$, P.Thirunavukkarasu ${ }^{2}$ \\ ${ }^{1}$ Research Scholar, Bharathiar University, Coimbatore, India. ${ }^{2}$ Associate Professor, Dept of Electronics, \\ SRMV College, Coimbatore, India.
}

\begin{abstract}
The proliferation of electronic devices in the automotive world has caused electromagnetic interference (EMI) and radio frequency interference (RFI) to become important concerns. Although all electronics emit magnetic and electrical energy, if this energy unintentionally interacts with another device and causes it to malfunction, then it is considered interference. EMI shielding has become a more significant issue due to the increased use of plastic housings for electronic equipment. Most plastics are insulators, so electromagnetic waves can pass through them freely and conductive barriers must be applied as shields to block the waves. To provide shielding, the plastic housing is coated with a conductive layer or the housing itself is made conductive. The coating method is much more common because it is easier to accomplish and is less dependent on the quality of the housing being shielded. In order to gauge the effectiveness of a shielding project, a specific set of tests and measurement standards can be applied to a shielded device or enclosure.
\end{abstract}

Index Terms: EMI, Cables, Shield Application, Measurements methods

\section{Introduction}

The auto industry's increased use of on-board electronics for guidance, entertainment and safety control systems. It has created substantial integrated circuit and system-on-chip (SoC) based design challenges to deliver energy and cost-efficient - yet highly reliable - electronics systems. As the use of on-board electronics in vehicles rises, power management plays a more critical role in automobile reliability. Increase of the internal components mid power density in a system, and many high speed digital electronic circuit without a shielding enclosure, even many interior and external interference sources are introduced so that the whole system applied electromagnetic environment becomes very serious. Electromagnetic shielding technique is one of the effective ways to control electromagnetic radiation from electronic equipment.

A shield is a metallic partition placed between two regions of space. It is used to control the propagation of electric and magnetic fields from one of the regions to the other. From an overall systems point of view, shielding the noise source is more efficient than shielding the receptor. However, there are cases where the source most be allowed to radiate and the shielding of individual receptors may be necessary. It is of little value to make a shield, no matter how well designed, and then allows electromagnetic energy to enter the enclosure by an alternative path such as cable penetrations.

\section{Cables}

In an automotive system, all the sensors and other actuators where connected to the electronics control modules via cable harnesses. These cable harnesses will be routed via so many noise sources presented in the system. So, these cables will pick up noise on one side of the shield and conduct it to the other side, where it will be re-radiated. In order to maintain the integrity of the shield enclosure, noise voltage should be filtered from all cables that penetrate the shield. This applies to power cables as well as signal cables. Cable shields that penetrate a shielded enclosure must be bonded to that enclosure in order to prevent noise coupling across the boundary.

\section{Shield Application}

Shields may be used to contain electromagnetic fields, if the shield surrounds the noise source as shown fig-1.

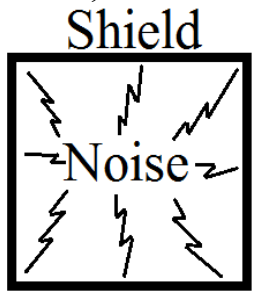


Fig-1. Shield application where a noise source is generated internal

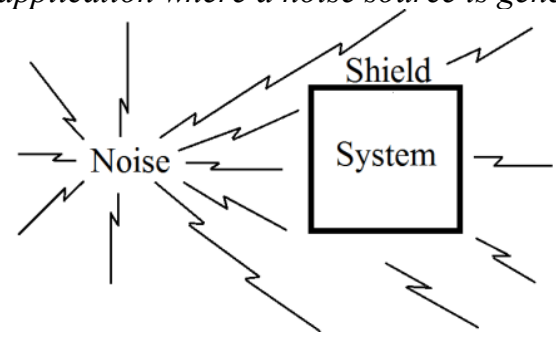

Fig-2. Shield application where interference is prevented by placing a shield around a receptor to prevent noise penetration.

This configuration provides protection for all susceptible equipment located outside the shied (assumption: no external fields). A shield may also be used to keep electromagnetic radiation out of region, as shown in fig- 2 . This provides protection only for the specific equipment contained within the shield (assumption: no system generated internal fields).

\section{Fields types}

The characteristics of a field are determined by the source, the media surrounding the source, and the distance between the source and the point of observation. At a point close to the source, the field properties are determined primarily by the source characteristics. Basically noises filed are classified in to two types, near fields \& far fields. Far from the source, the properties of the filed depend mainly on the medium through which the field is propagating. Therefore the space surrounding a source of radiation can be broken into two regions, as shown in fig-3.

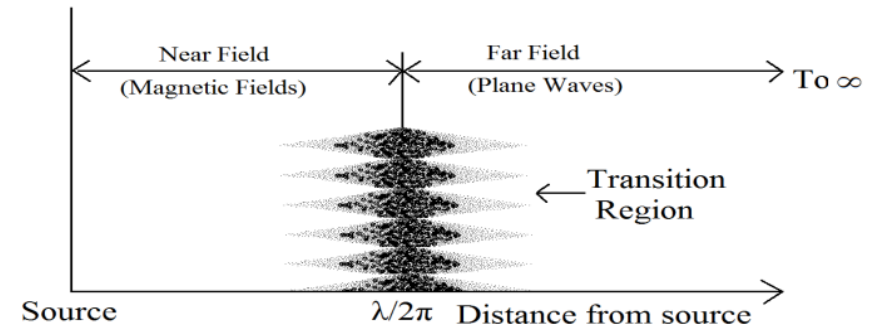

Fig-3. Field character depends on the distance from the source

Close to the source is the near, or induction, filed. At a distance greater than the wavelength $(\lambda)$ divided by $2 \pi$ is the far, or radiation, field. The region around $\lambda / 2 \pi$ is the transition region between the near and far fields.

\section{Wave Impedance}

The ratio of the electric filed $(E)$ to the magnetic field $(H)$ is the wave impedance. In the far field this ratio $\mathrm{E} / \mathrm{H}$ equals the characteristic impedance of the medium. Example: $E / H=Z_{0}=377 \Omega$ for free space. In the near filed the ratio is determined by the characteristics of the source and the distance from the source to where the field is observed. If the source has high current and low voltage $(E / H<377)$, the near filed is predominantly magnetic. Conversely, if the source has low current and high voltage $(E / H>377)$, the near filed is predominantly electric.

For a rod or straight wire antenna, the source impedance is high. The wave impedance near the antenna predominantly an electric filed is also high. As distance is increased, the electric field loses some of its intensity as it generates a complementary magnetic field.

In the near field the magnetic field attenuates at a rate of $(1 / \mathrm{r})^{3}$, whereas the electric field attenuates at a rate of $(1 / r)^{2}$. Thus the wave impedance from a straight wire antenna decreases with distance and asymptotically approaches the impedance of free space in the far filed, as shown fig-4. For a predominantly magnetic field such as product by a loop antenna the wave impedance near the antenna is low.

As the distance from the source increases, the magnetic field attenuates at a rate of $(1 / \mathrm{r}){ }^{3}$ and the electric fields attenuates at a rate of $(1 / \mathrm{r})^{2}$. The wave impedance therefore increases with distance and approaches that of free space at a distance of $\lambda / 2 \pi$. In the far filed both the electric and magnetic fields attenuate at a rate of $1 / \mathrm{r}$. 


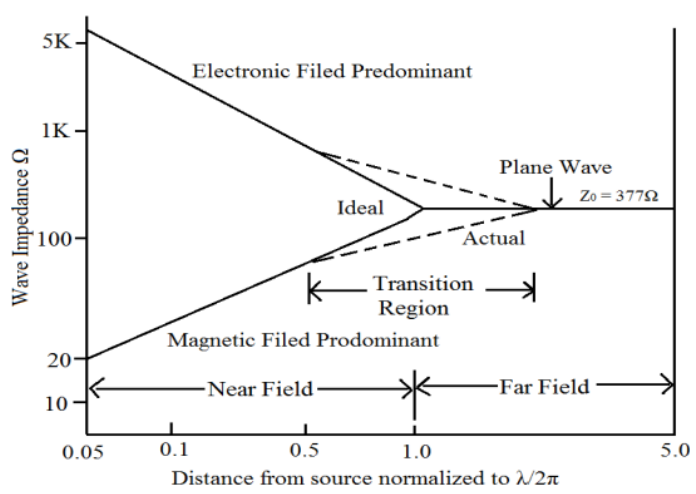

Fig-4. Wave impedance depends on the distance from the source and filed

In the near filed the electric and magnetic fields must be considered separately, since the ratio of the two is not constant. In the far field, however, they combine to form a plane wave having an impedance of $377 \Omega$. Therefore, when plan wave (also called a radiated field) are discussed, they are assumed to be in the far filed. When individual electric and magnetic fields (also called as induction field) are discussed they are assumed to be in the near field.

\section{Wave impedances characteristic}

We can take these constants to find the impedances

\begin{tabular}{|c|c|c|}
\hline Permeability & $\mu$ & $4 \pi \times 10^{-7} \mathrm{H} / \mathrm{m}$ for free space \\
\hline Dielectric constant & $\epsilon$ & $8.85 \times 10^{-12} \mathrm{~F} / \mathrm{m}$ for free space \\
\hline Conductivity & $\sigma$ & $5.82 \times 10^{7} \mathrm{~m} \Omega / \mathrm{m}$ for free copper \\
\hline
\end{tabular}

For any electromagnetic wave, the wave impedance is defined as

$$
R_{W}=\frac{E}{H} \quad \text {-------- } 1
$$

The characteristic impedance of a medium is defined by the following expression

$$
R_{W}=\sqrt{\frac{j \omega \mu}{\sigma+j \omega \epsilon}}
$$

In the case of a plane wave in the far field, $Z_{0}$ is also equal to the wave impedance $Z_{w}$. For insulators $(\sigma<<j \omega \epsilon)$ the characteristic impedance is independent of frequency and becomes

$$
Z_{0}=\sqrt{\frac{\mu}{\epsilon}}
$$

For free space $Z_{0}$ equals $377 \Omega$. In the case of conductors $(\sigma>>j \omega \epsilon)$, the characteristic impedance is called the shield impedance $Z_{\mathrm{S}}$ and becomes

$$
\begin{aligned}
& Z_{S}=\sqrt{\frac{j \omega \mu}{\sigma}}=\sqrt{\frac{\omega \mu}{2 \sigma}}=(1+j) \text {----- } 4 \\
& \left|Z_{S}\right|=\sqrt{\frac{\omega \mu}{\sigma}}
\end{aligned}
$$

For copper at $1 \mathrm{kHz},\left|Z_{S}\right|$ equals $1.16 \times 10^{-5} \Omega$. Substituting numerical values for the constants of eq.5 gives the following results 
For copper,

$$
\left|Z_{S}\right|=3.68 \times 10^{-7} \sqrt{f}
$$

For aluminum,

$$
\left|Z_{S}\right|=4.71 X 10^{-7} \sqrt{f}
$$

For steel,

$$
\left|Z_{S}\right|=3.68 \times 10^{-5} \sqrt{f}
$$

For any conductor, in general

$$
\left|Z_{S}\right|=3.68 \times 10^{-7} \sqrt{\frac{\mu_{r}}{\sigma_{r}}} \sqrt{f} \quad \text {-------- } 9
$$

\section{Shielding Effectiveness}

Shielding effectiveness can be determined by analyzing the problem in either of two ways.

1. Analyzing using circuit theory

2. Analyzing using field theory

In the circuit theory approach the noise fields induce currents in the shields, and these currents in turn generate additional fields that tend to cancel the original fields in certain regions. This effect is shown in fig-4. Also we will adopt the more fundamental filed theory approach. Shielding can be specified in term of the reduction in magnetic or electric filed strength caused by the shield. It is convenient to express this shielding effectiveness in units of decibels $(\mathrm{dB})$. Use of $\mathrm{dB}$ then permits the shielding produced by various effects to be added to obtain the total shielding. Shielding effectiveness $(S)$ is defined for electric fields as

$$
S=20 \log \frac{E_{0}}{E_{1}} d B
$$

And for magnetic fields as

$$
S=20 \log \frac{H_{0}}{H_{1}} d B
$$

In this preceding equations, $E_{0}\left(H_{0}\right)$ is the incident filed strength, and $E_{1}\left(H_{1}\right)$ is the field strength of the transmitted wave as it emerges from the shield. In the design of a shielded enclosure, there are two prime considerations.

1. Shielding effectiveness of the shield material

2. Shielding effectiveness due to discontinuities and holes in the shield

Shielding effectiveness varies with frequency, geometry of shield, position within the shield where the field is measured, type of filed being attenuated, direction of incidence, and polarization. The total shielding effectiveness of a material is equal to the sum of the absorption loss $(A)$ and reflection loss $(R)$ and correction factor $(B)$ to account for multiple reflections in this shield in thin shields. Total shielding effectiveness therefore can be written as

$S=A+R+B \mathrm{~dB}$ 12 
All the terms in eq.12 must be expressed in dB. The multiple reflection factor $B$ can be neglected if the absorption loss $A$ is much greater than B. From a practical point of view $B$ can also be neglected for electric filed and plane waves.

\section{Shielding Effectiveness Measurements (far field)}

The concept of plane-wave shielding effectiveness is convenient because it is a function of only the material properties and thickness of a shielding material. Attempts to measure the plane-wave shielding effectiveness generally involve launching a guided TEM wave in a coaxial test fixture containing a sample of the material, as illustrated in Fig. 5.

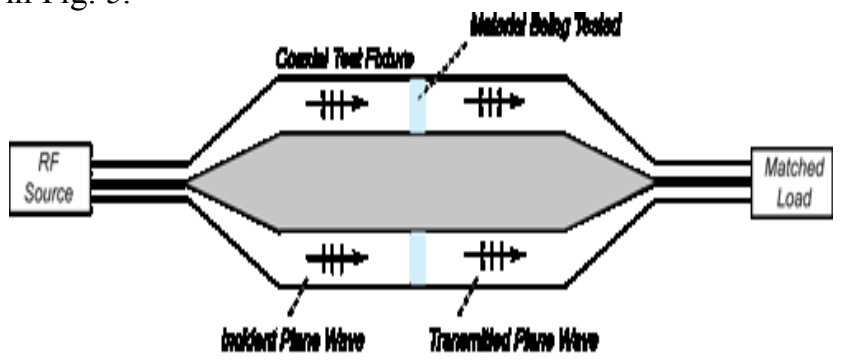

Fig- 5. Shielding Effectiveness Test Fixture

The transmission line structure has specific characteristic impedance (usually $50 \mathrm{ohms}$ ). The cross-sectional dimensions are scaled up in the mid-section of the test fixture in order to accommodate a reasonably sized material sample, which is disk-shaped with a hole in the center.

\section{Summary}

We have to take addition care while selecting the shielding enclosure for the automotive electronics. All cables entering a shielded enclosure should be filtered. Shielded cables entering a shielded enclosure should have their shields boned to the enclosure. Reflection loss is very large for electric fields and plan waves and normally it will be small for low frequency magnetic fields. We have to use magnetic materials to shield against low frequency magnetic fields also, a good conductor to shield against electric fields, plane wave, and high frequency magnetic fields.

\section{Reference}

[1] Burgoon, J.R., Jr “Fundamentals od Electrical Shield Design.” Insulation/Circuits, August 1970.

[2] Cook, D.V. "RFI Suppression, Part I" Electromechical Design, Vol 11 November 1967.

[3] Cowdel, R.B. "Nomographs Simplify Calculations of Magnetic Shielding Effectiveness" EDN Vol.17 September 1, 1972.

[4] Ficchi, R.O. Electrical Interference. Hayden Book Co., New York 1964.

[5] Frederick Research Corp. Handbook on Radio Frequency Interference. Vol.3. Frederick Research Copr., Wheaton.

[6] Gerteisen, S.R. "Conductive Thermoplastics Meet Shielding Demands" Design News, January 21, 1985.

[7] Grant, P.M. "Design Guidelines to EMI Shielding Windows" Tecknit EMI Shielding Products, Cranford, N J.

[8] Hayt, W.H.,Jr. Engineering Electromagnetics, $3^{\text {rd }}$ edition. McGraw-Hill, New York. 